\title{
Effect of the Magnetic Force on Ferrite Pendulum Oscillation Parameters: Parametric Analysis on Ferrite Pendulum
}

\author{
Rusli Adi $^{1 *}$, Moh Toifur ${ }^{1}$, Ishafit ${ }^{1}$ and Rosly Jaafar ${ }^{2}$ \\ ${ }^{1}$ Master Program of Physics Education, Universitas Ahmad Dahlan \\ Jl. Pramuka No. 42, Yogyakarta, Daerah Istimewa Yogyakarta, Indonesia \\ ${ }^{2}$ Department of Physics Education, Universiti Pendidikan Sultan Idris \\ Tanjong Malim, Perak, Malaysia \\ *Email: rusliadyfisika@gmail.com
}

\begin{abstract}
Article Info
ABSTRACT

Article History

Received Feb 27, 2020

Revised Jun 19, 2020

Accepted Jun 22, 2020

Keywords:

Damping coefficient

Ferrite pendulum

Magnetic force

Swing pendulum

The magnitude of the damping force of the mathematical pendulum swinging on a medium is usually proportional to the speed of the pendulum. In this research, the pendulum oscillation parameters oscillating on an air medium under the influence of a magnetic field of $1.8 \mathrm{G}$ will be investigated. In the initial stage, the effect of the magnetic force on the damping coefficient of ferrite pendulum oscillations with an initial deviation of 15 degrees observed. Furthermore, the study continued with varying the angle of deviation from 5 degrees to 25 degrees. The results of the data fitting amplitude (xi) at various swing times (ti) are using to analyze the effect of the angle of deviation on the maximum amplitude. The results showed that for the deviation angle of $15^{\circ}$ the coefficient of damping of the medium affected by the magnetic force was 0,0022 greater than the coefficient of air damping 0,00006 . It affects the amplitude, which decreases faster than the pendulum amplitude without the influence of magnetic force. Variation in the angle of deviation also affects the amplitude of the pendulum. In the deviation angle below 10, the pendulum motion is more influenced by the magnetic force, whereas in the deviation above 10 , the pendulum motion is more dominated by gravity.
\end{abstract}

This is an open access article under the $\mathrm{CC}-\mathrm{BY}-\mathrm{SA}$ license.

To cite this article:

R. Adi, M. Toifur, Ishafit, and R. Jaafar, "Effect of the Magnetic Force on Ferrite Pendulum Oscillation Parameters: Parametric Analysis on Ferrite Pendulum," Indones. Rev. Phys., vol. 3, no. 1, pp. 1-5, 2020.

\section{Introduction}

There are two types of vibrations, namely harmonized vibration and damped vibration. Damped oscillations consist of various kinds, namely dumped, overdamped, and underdamped. The type of dropped occurs if there is attenuation, over dumped occurs if the attenuation is maximum so that objects cannot vibrate anymore, underdamped vibrations arise under the influence of force.

Swing pendulum vibrations with weights made of ferrite material [1] under the influence of permanent magnetic fields [2], [3], [4] are examples of underdamped vibrations. This type of swing is seen as a rather specific swing because the pendulum vibration is limited by the influence of the coercive force, which is a combination of magnetic attraction and magnetic repulsion forces. The spectacle occurs when the pendulum polarity is opposite to the permanent magnet polarity, and the repulsion occurs when the pendulum polarity is the same as the permanent magnet. The pull and push forces and the force of gravity produce a swing that is only slightly damp. It can be observed from the amplitude of the oscillation that changes only slightly so that the swing pattern is still like a harmonic vibration [5]. This condition runs in a few moments. When the gravitational run is no longer able to compensate the attractive and repulsive magnetic forces, the magnetic force is more dominant so that the swing is no longer harmonious. The amplitude of the oscillation decreases dramatically and finally stops. Therefore, in this study, the solution of differential equations with dumping force derived from the interaction between the ferromagnetic pendulum and the permanent magnet. By varying the angle of displacement of the oscillator then it can be seen its effect on the maximum deviation after there is a dumping magnetic force. 


\section{Theoretical \\ Damped oscillation}

Vibration is a back and forth repetitive movement that repeats through the equilibrium point [6], [7]. The harmonic vibration equation is given [8] in the eq. (1):

$m \frac{d^{2} x}{d t^{2}}+k x=0$

Theoretically on harmonious vibrations, the pendulum will oscillate with a fixed amplitude. But in reality, the object will stop oscillating after a particular time due to air attenuation [9] and other things such as magnetic fields. The equation for damped swing where the damping force is proportional to the swing speed [10] is

$\vec{F}_{a b}=\frac{3 \mu_{0} m_{a} m_{b}}{4 \pi r^{4}}\left\{\hat{r}\left(\hat{m}_{a} \cdot \hat{m}_{b}\right)+\hat{m}_{a}\left(\hat{r} \cdot \hat{m}_{b}\right)+\hat{m}_{b}\left(\hat{r} \cdot \hat{m}_{a}\right)-5 \hat{r}\left(\hat{r} \cdot \hat{m}_{a}\right)\left(\hat{r} \cdot \hat{m}_{b}\right)\right\}$

Where $m_{\mathrm{a}}$ and $m_{\mathrm{b}}$ is the pendulum magnetic moment of $a$ and $b$, and $\hat{m}_{a} \hat{m}_{b}$ the unit magnetic moment $m_{a}$ and $m_{\mathrm{b}}, r$ the distance from $a$ to $b$, and $\hat{r}$ the position vector $a$ from $b$. The magnitude interaction force between $a$ and $b$ is dependent on the interval [16] and the orientation of the magnetic moment of the two magnets.

\section{Ferrite rod pendulum swing}

Figure 1 shows the ferrite rod an as a pendulum swinging under the influence of a permanent magnet $b$.

At the beginning of pendulum, a deviated at an angle $\theta$. When the pendulum is released, the gravitational force causes the pendulum to swing near the magnetic pole $b$, for example, the North pole (U). At the same time, the ferrite pendulum is magnetized by the magnetic field $\mathrm{b}$ so that the magnetic dipole moments a near the North pole $b$ become the south pole (S). In this condition, the driving force comes from 2 sources, namely the gravitational force and the magnetic interaction force. Assume that the gravity force is greater than the magnetic force.

Furthermore, when a is near the centre of mass of the permanent magnet $b$, the status of pendulum a leaves the north pole of magnet $b$ and goes to the south pole of magnet $b$. In this case, the pendulum is affected by both permanent magnetic poles $b$. Towards the north pole of stable magnet $b$, the pendulum is pulling, and this pull is inhibiting motion. Once it is released from the magnetic north pole, the pendulum is induced by the magnetic South pole so that its polarity reverses to become the

$$
x(t)=A e^{-\lambda} \sin (\omega t-\theta)+\frac{\omega^{2} A}{\sqrt{\left(\omega_{0}^{2}-\omega^{2}\right)^{2}+4 \omega^{2} \gamma^{2}}} \cos (\omega t-\theta)
$$

$m \frac{d^{2} x}{d t^{2}}+b \frac{d x}{d t}+k x=0$

Where $x$ displacement, $m$ pendulum mass, $b$ damping coefficient, and $k$ string constants. For swing under the influence of the magnetic force, there is a damped force that works period so that it will affect the oscillation frequency and maximum deviation [11]. For a driving force, $F \cos \omega t$ the [12] eq. (3) can be written as

The magnitude of the interaction force between the ferromagnetic pendulum (a) and the permanent magnet (b) at distance $r$ is expressed by eq. (4), [13], [14], [15]. $m \frac{d^{2} x}{d t^{2}}+b \frac{d x}{d t}+k x=F \cos \omega t$

north pole due to the pull of the magnetic north pole on the south pole of the pendulum deviation to be reduced. The same pendulum motion occurs in the opposite direction so that the pendulum deviation decreases over time and finally stopped.

It should be noted that when the pendulum is near the magnet, the swing becomes less regular, which is slightly away from the line. It is because of the magnetic repulsion force on the pendulum when the polarity of the pendulum is the same as the magnetic polarity. By viewing the magnetic force by magnet $b$ as a damped force, the solution eq. (3) is stated by [17]:

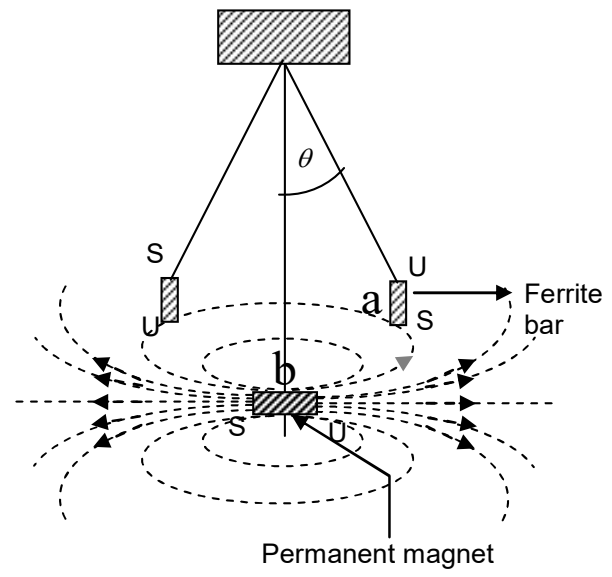

Figure 1. The pendulum system under the influence of magnetic forces. 
For frequency of damped force $\omega$ that equals to natural rate $\omega_{0}$ then

$$
x(t)=A e^{-\gamma t} \sin (\omega t-\theta)+\frac{\omega A}{2 \gamma} \cos (\omega t-\theta)
$$

Where $A$ is oscillation amplitude. The quantities $\omega$ and $\theta$ in the sin term differ from those in the cos term, except under certain conditions.

\section{Method}

\section{Tools and materials}

The material used in this study consisted of ferrite rods with a mass of $0.0258 \mathrm{~kg}$, a diameter of $0.955 \mathrm{~cm}$, length of $3 \mathrm{~cm}$, a thread of $1 \mathrm{~m}$, rectangular neodymium magnets with a size of 2 $\mathrm{cm}^{\prime} 5 \mathrm{~cm}^{\prime} 0.2 \mathrm{~mm}$ of 2 pieces. This magnet has an 8 $\mathrm{mT}$ measured at a distance of $1 \mathrm{~cm}$ from it one pole. The tools needed include a protractor to measure the angle of deviation, a $50 \mathrm{~cm}$ support rod, a video to record the movement of the pendulum, tracker software to obtain pendulum position data at any time.

\section{Data collection}

The ferrite pendulum is hanging on a $38 \mathrm{~cm}$ long string then placed on a $40.5 \mathrm{~cm}$ high statif. Neodymium magnets as permanent magnets $0.5 \mathrm{~cm}$ thick are mounted on the base so that the distance from the end of the ferrite pendulum to the permanent magnet is $2 \mathrm{~cm}$. Furthermore, the ferrite pendulum is deviated at an angle of 5 degrees and then released. The oscillation of the pendulum is recorded with the video until the pendulum stops swinging. This experiment was carried out in a confined space so that the disturbance from the environment can be minimized.

\section{Data analysis}

From the pendulum swing video tracking the pendulum data set of the position of a pendulum at any time $\left(t_{i}, y_{i}\right)$ is obtained. The data is then fitted according to the eq. (7):

$$
X=A e^{-D t} \sin (\omega t+\theta)+E \cos (\omega t+\theta)
$$

Where $X$ is pendulum deviation, $A, \omega, \theta$, and $D$ are fitting constants. From this data, fitting also obtained RMSE and $\mathrm{R}^{2}$ values. A right $\mathrm{R}^{2}$ is more than 0.95 . That the value of $\mathrm{R}^{2}$ is still far from 0.95 so that the fitting parameters are corrected with solver parameters software.

\section{Results and Discussion}

\section{Damping coefficient of the magnetic field}

Figure 2 shows an example of the appearance of the ferrite pendulum tracking results for an angle of 15 degrees without the influence of a magnetic field. Data sampling was performed at every $0.02 \mathrm{~s}$. We are tracking data in the form of time as $\mathrm{x}$ and position as $y$. From this tracking data, then an $x(t)$ curve is drawn. The same thing is done for the swing with the influence of the magnetic field. The $x(t)$ curve together shown in Figure 2.

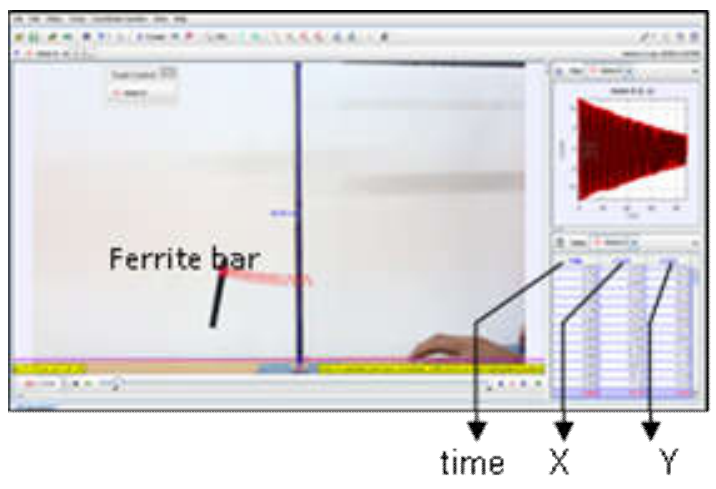

Figure 2. Display of the tracking process on ferrite pendulum swing to get it to a position at any time.

Figure 3 shows the curved pendulum ferrite pendulum tracking results for swing without the damping, with air attenuation and with the damping from permanent magnets.

From Figure 3, it appears that in general, the longer the oscillation, the deviation is decreasing. For oscillations without damping, the amplitude of the pendulum does not change for oscillations with air attenuation the variance of the amplitude decreases. Table 1 shows the oscillation parameters of the ferrite pendulum fittings.

From Table 1 it appears that the deviations in the three types of vibrations were initially the same, namely $17.75 \mathrm{~cm}$. But after swinging, the pendulum influenced by magnetic force tends to be $(A+E)$ smaller than the pendulum with air attenuation or without damping that is A. In contrast, the damping coefficient $\gamma$ of the three types are different. From the value of $\gamma$, it appears that the swing with attenuation from the magnetic force has $\gamma=0,022$ higher than the swing without the magnetic force that is $\gamma=0.00006$. It shows the role of the magnetic force on the ferrite pendulum swing, especially when leaving the equilibrium position, the pull force between the ferrite pendulum and the magnet can inhibit the pendulum's motion so that the deviation becomes reduced. However, the pendulum still swings at the same frequency on all swing types that is 4.61 $\mathrm{rad} / \mathrm{s}$. 


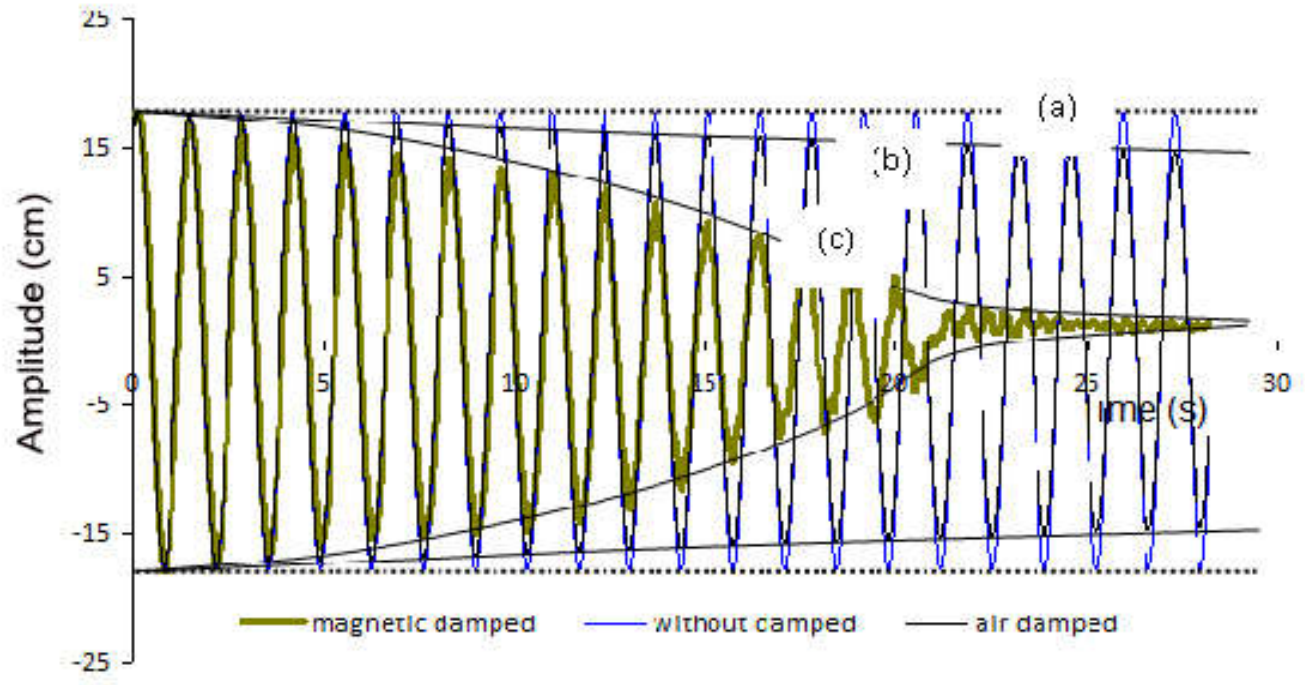

Figure 3. Swing of the ferrite pendulum at an angle of deviation of 15 degrees (a) without damping, (b) with air attenuation, c) with magnetic field attenuation.

Table 1. Fitting constants in various swing conditions

\begin{tabular}{lll}
\hline Type of oscillation & Equation of pendulum Position & Fitting constants \\
\hline & & $A=17.75 \mathrm{~cm}$ \\
Undamping & $y=A e \sin (\omega t+\theta)$ & $\omega=4.61 \mathrm{rad} / \mathrm{s}$ \\
(theoretically) & $\gamma=0$ \\
& $\theta=25^{\circ}$ \\
& $A=17.75 \mathrm{~cm}$ \\
Air damping & $\omega=4 e^{-\gamma t} \sin (\omega t+\theta)$ & $\omega=4 \mathrm{rad} / \mathrm{s}$ \\
& $\gamma=0.00006$ \\
& $\theta=25^{\circ}$ \\
Magnetic force & $A=17.75 \mathrm{~cm}$ \\
damping & $y=A e^{-\gamma t} \sin (\omega t+\theta)+E \cos (\omega t+\theta)$ & $E=0.13 \mathrm{~cm}$ \\
& $\omega=6.61 \mathrm{rad} / \mathrm{s}$ \\
& & $\gamma=0.02200$ \\
\end{tabular}

\section{Effect of angle of deviation on the amplitude}

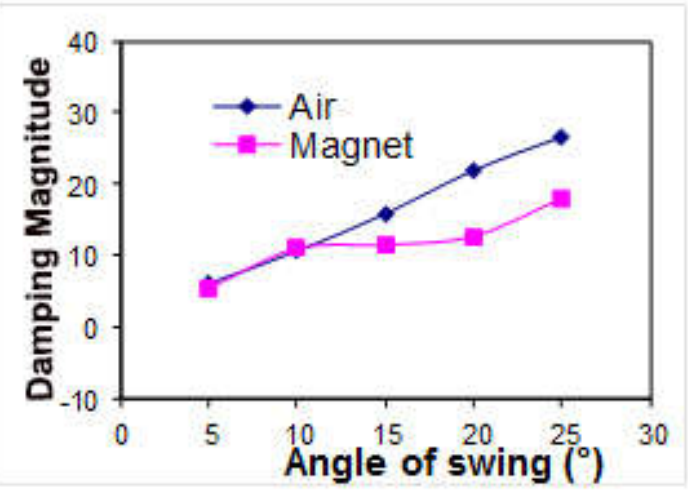

Figure 4. The amplitude of ferrite pendulum at various deviation angles.

From Figure 4, the damping magnitude for air and magnet are displayed at various angles of pendulum deviation. At an angle of 5 degrees to 10 degrees, the pendulum amplitude curve for magnetic attenuation coincides with the pendulum amplitude with air attenuation. At small angles, the gravitational force that moves the pendulum toward the horizontal is almost not present so that the magnetic force dominates the pendulum driving force. Assume that the pendulum moves from the right to the left. When the position pendulum at slightly the right of the magnet, the pendulum pulled to the left by the magnet. Once it passes through the equilibrium, the magnetic induction process occurs so that the polarity of ferrite pendulum reversed. As a result, the pendulum is pulled back from the left position to the equilibrium point. This movement only occurs a few times, and after that, the pendulum will stay in the place closest to the magnet.

For deviation angles above 10 degrees, the amplitude of the ferrite pendulum with a magnetic field damper is smaller than the amplitude of the 
ferrite pendulum with an air damper. At an angle of deviation above 10 degrees, the gravitational force can play a more significant role in moving the pendulum. In contrast, the magnetic force is still small because the magnetic distance to the pendulum is further away. When the pendulum released to the equilibrium point, the pendulum moves quickly because of the influence of the gravitational force, and this driving force increased when the pendulum approaches the magnet because the arising the magnetic force. After the pendulum passes the equilibrium point, the polarity changes due to magnetic induction, and this inhibits the pendulum's motion. As a result, the amplitude is reduced. The same mechanism occurs in the swing in the opposite direction until the pendulum stops. Thus at an angle of more than 10 degrees, the presence of a magnetic field can reduce the amplitude of the pendulum swing compared to if there is no magnetic field.

\section{Conclusion}

The ferrite pendulum that swings under the influence of the magnetic force has a characteristic in its swing profile. The damping coefficient is higher than the air damping coefficient, so the amplitude decreases so that the pendulum stops faster. The air attenuation coefficient is 0.00006 , while the magnetic damping coefficient is 0.0022 . At an angle deviation, less than 10 degrees the force motion in the pendulum more dominated by the magnetic force whereas in the deviation above 10 degrees the force of gravity more dominates the force of movement. But the swing is inhibited by the magnetic force so that the amplitude decreases.

\section{Reference}

[1] G. Khomeriki, "Parametric Resonance Induced Chaos in the Magnetic Damped Driven Pendulum," Phys. Lett. A, vol. 380, no. 31-32, pp. 2382-2385, 2016.

[2] J. Sanada, K. Furuno, H. Hirakawa, and T. Kato, "Application of the Magnetic Fluid as A Detector for Changing the Magnetic Field Application of the Magnetic Fluid as A Detector for Changing the Magnetic Field," Int. Conf. "Cognitive Robot., vol. 363, no. 012023, pp. 6-12, 2018.

[3] B. M. Teo, D. J. Young, and X. J. Loh, "Magnetic Anisotropic Particles: Toward Remotely Actuated Applications," Part. Part. Syst. Charact., pp. 1-6,
2016.

[4] K. A. Kumar, S. F. Ali, and A. Arockiarajan, "Magneto-elastic Oscillator: Modeling and Analysis with Nonlinear Magnetic Interaction," $J$. Sound Vib., vol. 393, pp. 265-284, 2017.

[5] A. I. Shumaev and Z. A. Maizelis, "Distribution Functions of Argumental Oscillations of the Duboshinskiy Pendulum," Phys. Chem. Process., vol. 154902, no. 121, pp. 1-10, 2017.

[6] Y. Uzun, E. Kurt, and H. H. Kurt, "Explorations of Displacement and Velocity Nonlinearities and Their Effects to Power of a Magnetically-Excited Piezoelectric Pendulum Sensors and Actuators A: Physical Explorations of displacement and velocity nonlinearities and their effects to power of," Sensors Actuators A. Phys., vol. 224, pp. 119-130, 2018.

[7] K. Polczyński, A. Wijata, and J. Awrejcewicz, "Theoretical and Numerical Analysis of Different Modes in a System of a 'Kicked' Magnetic Pendulum," Theor. Approaches Non-Linear Dyn. Syst., pp. 431-440, 2019.

[8] R. A. Serway and J. W. J. Jewett, Physics for Scientists and Engineers with Modern Phy. United States: Thomson, 2008.

[9] E. I. Butikov, "Spring Pendulum with Dry and Viscous Damping," Commun. Nonlinear Sci. Numer. Simul., vol. 20, no. 1, pp. 298-315, 2015.

[10] G. D. Quiroga and Ospina-Henao, "Dynamics of Damped Oscillations: Physical Pendulum," EJP, pp. 1-5, 2017.

[11] G. L. Baker and J. A. Blackburn, "The Pendulum: a Case Study in Physics," in The Pendulum: A Case Study in Physics, vol. 43, no. 06, New York: Oxford University Press, 2006, pp. 43-3440-433440

[12] D. C. Giancoli, Physics for Scientists and Engineers with Modern Physics, vol. 26, no. 4. New Jersey: Pearson Prentice Hall, 1988.

[13] Y. Luo, W. Fan, C. Feng, S. Wang, and Y. Wang, "Subharmonic Frequency Response in a Magnetic Pendulum," Am. J. Phys., vol. 88, no. 2, pp. 115123, 2020.

[14] K. W. Yung, P. B. Landecker, and D. D. Villani, "An Analytic Solution for the Force Between Two Magnetic Dipoles," Magn. Electr. Sep., vol. 9, no. 1, pp. 39-52, 1998.

[15] B. F. Edwards, D. M. Riffe, J.-Y. Ji, and W. A. Booth, "Interactions Between Uniformly Magnetized Spheres," Am. J. Phys., vol. 85, no. 2, pp. 130-134, 2017.

[16] A. A. Khajetoorians et al., "Tailoring the Chiral Magnetic Interaction Between Two Individual Atoms," Nat. Commun., vol. 7, pp. 1-8, 2016.

[17] L. P. Pook, Understanding Pendulum. Springer, 2011. 